\title{
Observation-based Analysis of Ozone Production Sensitivity for Two Persistent Ozone Episodes in Guangdong, China
}

Kaixiang Song ${ }^{1}$, Run Liu ${ }^{1,2}$, Yu Wang ${ }^{1,2}$, Tao Liu ${ }^{1}$, Liyan Wei ${ }^{1}$, Yanxing Wu ${ }^{1}$, Junyu Zheng ${ }^{1,2}$, Boguang Wang ${ }^{1,2}$, Shaw Chen Liu ${ }^{1,2}$

$5 \quad{ }^{1}$ Institute for Environmental and Climate Research, Jinan University, Guangzhou, 511443, China

${ }^{2}$ Guangdong-Hongkong-Macau Joint Laboratory of Collaborative Innovation for Environmental Quality, Guangzhou, 511443, China

Correspondence to: Run Liu (liurun@jnu.edu.cn), Shaw Chen Liu (shawliu@jnu.edu.cn) 


\section{Supplementary Tables}

10 Table S1. Ratios of VOC species to $\mathrm{CO}$ and the corresponding reaction rate constants of individual $\mathrm{VOC}_{\mathrm{s}}$ with $\mathrm{OH}$ (Huang et al., 2021).

\begin{tabular}{|c|c|c|c|c|}
\hline & $\mathrm{VOC}_{\mathrm{S}} / \mathrm{CO}$ & $\mathrm{VOC}_{\mathrm{S}} / \mathrm{VOC}_{\mathrm{S}}$ (total) & molecular mass & $10^{-12} \times \mathrm{K}_{\mathrm{OH}}$ \\
\hline Methylbenzene & 0.01266 & 0.0634 & 92 & 5.63 \\
\hline M/P-xylene & 0.00965 & 0.04836 & 106 & 18.9 \\
\hline Formaldehyde & 0.00900 & 0.0451 & 30 & 9.37 \\
\hline Ethylbenzene & 0.00771 & 0.03863 & 106 & 7 \\
\hline N-hexane & 0.00582 & 0.0292 & 86 & 5.2 \\
\hline Ethylene & 0.00544 & 0.02728 & 28 & 8.52 \\
\hline Acrylic & 0.00479 & 0.02399 & 42 & 26.3 \\
\hline Acetone & 0.00475 & 0.02379 & 58 & 0.17 \\
\hline Benzene & 0.00432 & 0.02166 & 78 & 1.22 \\
\hline O-xylene & 0.00407 & 0.02038 & 106 & 13.6 \\
\hline Ethanol & 0.00405 & 0.02032 & 46 & 3.2 \\
\hline Ethane & 0.00397 & 0.01992 & 30 & 0.26 \\
\hline Butanone & 0.00331 & 0.01662 & 72 & 1.22 \\
\hline Methanol & 0.00326 & 0.01635 & 32 & 0.94 \\
\hline N-butane & 0.00319 & 0.01601 & 58 & 2.36 \\
\hline Cyclohexane & 0.00300 & 0.01504 & 84 & 6.97 \\
\hline Propane & 0.00297 & 0.01489 & 44 & 1.09 \\
\hline N-pentane & 0.00289 & 0.01451 & 72 & 3.8 \\
\hline 2-methylpentane & 0.00274 & 0.01377 & 86 & 5.2 \\
\hline Acetaldehyde & 0.00272 & 0.01364 & 44 & 15 \\
\hline 1-butene & 0.00238 & 0.01193 & 56 & 31.4 \\
\hline 1,2,4-Trimethylbenzene & 0.00222 & 0.01114 & 120 & 32.5 \\
\hline M-xylene & 0.00152 & 0.00761 & 106 & 23.1 \\
\hline N-heptane & 0.00140 & 0.00706 & 100 & 6.76 \\
\hline 4-Methyl-2-pentanone & 0.00123 & 0.00616 & 100 & 13 \\
\hline N-dodecane & 0.00101 & 0.00508 & 170 & 13.2 \\
\hline 1,2,3-Trimethylbenzene & 0.00089 & 0.00448 & 120 & 32.7 \\
\hline 1,3,5-Trimethylbenzene & 0.00086 & 0.00431 & 120 & 56.7 \\
\hline N-octane & 0.00083 & 0.00419 & 114 & 8.11 \\
\hline 2,3-Dimethylbutane & 0.00080 & 0.00405 & 86 & 5.78 \\
\hline
\end{tabular}




\begin{tabular}{ccccc}
\hline Hexanal & 0.00075 & 0.00379 & 100 & 30 \\
Undecane & 0.00073 & 0.00367 & 156 & 12.3 \\
O-ethyl toluene & 0.00073 & 0.00367 & 120 & 11.9 \\
Trans-2-butene & 0.00070 & 0.00353 & 56 & 64 \\
N-decane & 0.00069 & 0.00346 & 142 & 11 \\
P-ethyl toluene & 0.00067 & 0.00336 & 120 & 11.8 \\
2,2,4-Trimethyl pentane & 0.00067 & 0.00335 & 114 & 3.34 \\
Total & 0.23547 & 0.6082 & $/$ & $/$ \\
\hline
\end{tabular}

